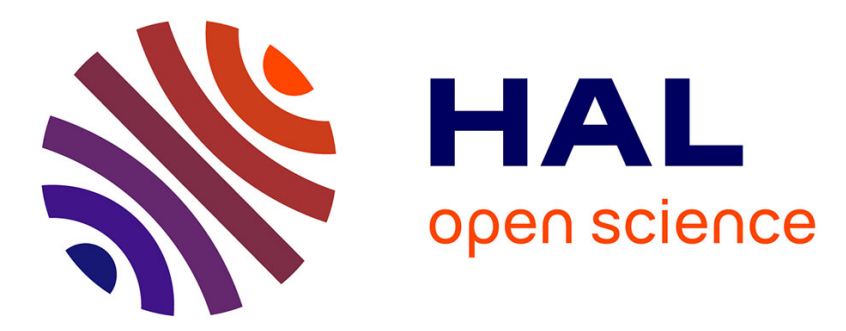

\title{
Commensurate-incommensurate transition with frozen impurities
}

\author{
J. Villain
}

\section{To cite this version:}

J. Villain. Commensurate-incommensurate transition with frozen impurities. Journal de Physique Lettres, 1982, 43 (15), pp.551-558. 10.1051/jphyslet:019820043015055100 . jpa-00232091

\section{HAL Id: jpa-00232091 https://hal.science/jpa-00232091}

Submitted on 1 Jan 1982

HAL is a multi-disciplinary open access archive for the deposit and dissemination of scientific research documents, whether they are published or not. The documents may come from teaching and research institutions in France or abroad, or from public or private research centers.
L'archive ouverte pluridisciplinaire HAL, est destinée au dépôt et à la diffusion de documents scientifiques de niveau recherche, publiés ou non, émanant des établissements d'enseignement et de recherche français ou étrangers, des laboratoires publics ou privés. 
Classification

Physics Abstracts

$64.70-61.70-68.20$

\title{
Commensurate-incommensurate transition with frozen impurities
}

\author{
J. Villain \\ DRF/G, Laboratoire de Diffraction Neutronique, \\ Centre d'Etudes Nucléaires de Grenoble, 85 X, 38041 Grenoble Cedex, France
}

(Reçu le 13 mai 1982, accepté le 15 juin 1982)

\begin{abstract}
Résumé. - Nous discutons un modèle de transition commensurable-incommensurable avec impuretés figées. $A D=3$ dimensions le déplacement du pic de Bragg varie linéairement avec le potentiel chimique ou la température près de la transition. A la dimension critique inférieure $D_{l}=2$ la phase commensurable est en principe toujours instable. On discute l'analogie avec le modèle d'Ising en champ aléatoire pour lequel $D_{l}$ serait égal à 3 d'après des travaux récents. On souligne qu'il est difficile d'appliquer la méthode des répliques.
\end{abstract}

\begin{abstract}
A model of commensurate-incommensurate transition with frozen impurities is discussed. In $D=3$ dimensions the Bragg peak shift varies linearly with the chemical potential or temperature near the transition. At the lower critical dimension $D_{l}=2$, the commensurate phase is, strictly speaking, always instable. There is some analogy with an Ising model in random fields, where $D_{l}$ is claimed to be 3 according to recent work. The replica trick is shown to be difficult to apply.
\end{abstract}

1. The model. - According to a well-known picture [1-5], incommensurate phases can be represented, near the commensurate-incommensurate (C-I) transition, as a succession of nearly commensurate domains separated by " walls", also called " discommensurations " and " solitons ". In this note, we consider only the case of walls with a single easy direction parallel to a $d$-dimensional plane $\Pi_{d}$ (Fig. 1). $d+1=D$ is the space dimensionality. $D=3$ or 2 in real systems. A point $\mathbf{R}$ of the $D$-dimensional space may be written $\mathbf{R}=(\mathbf{r}, z)$, where $\mathbf{r}$ denotes one of the $N^{\perp}$ sites of a $d$-dimensional Bravais lattice in $\Pi_{d}$, and $z$ takes integer values between $-N^{\| / 2}$ and $N^{\| /} / 2 . z$ also denotes the axis perpendicular to $\Pi_{d}$.

A basic assumption of the model is that the parallel to $z$ at any site $\mathbf{r} \in \Pi_{d}$ is assumed to cut any wall (say, the $m^{\prime}$ th wall) at a single point $\left(\mathbf{r}, \zeta_{r m}\right)$. Physically, this implies that the wall stiffness is high. $\zeta_{r m}$ might be multivalued for two alternative reasons : i) if there are dislocations [6] ; ii) if both " light " and " heavy » walls [2] are present. The present model is always approximate, but the approximation may be very good.

The purpose of the present work is to study the effect of random, frozen impurities. Consider for instance (Fig. 2a) a Xe monolayer on the (110) face of $\mathrm{Cu}$ [7]. Xe atoms have to choose between two sublattices A, B. If a chemisorbed impurity is frozen at an A site, it probably favours the occupation of B sites by Xe atoms. Thus, if A domains are represented by an Ising spin $S_{R}=1$, and B domains by $S_{R}=-1$, frozen impurities are equivalent to a Hamiltonian

$$
\mathscr{H}=-\sum_{R} H_{R} S_{R}
$$



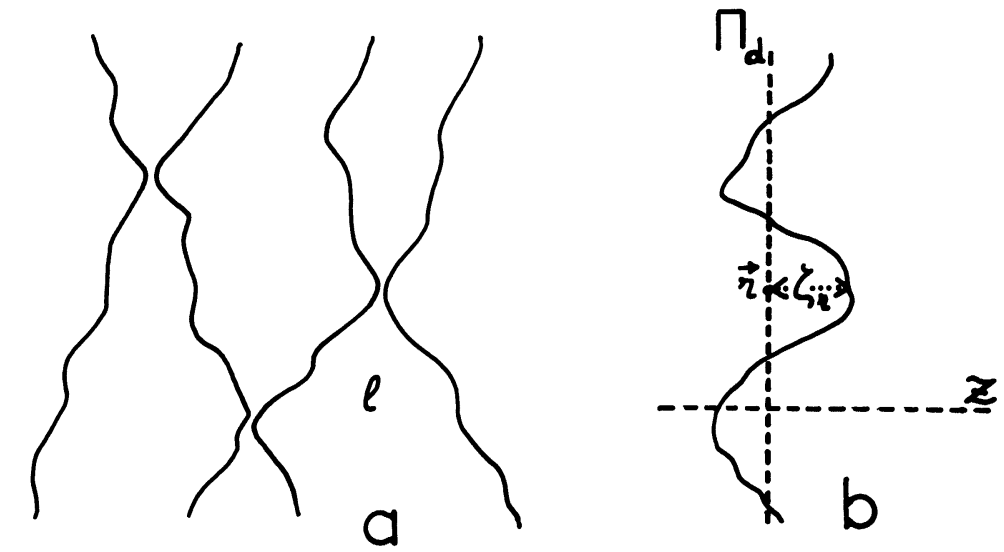

Fig. 1. $-a$ ) Array of walls in an anisotropic incommensurate system near the C-I transition, for $d=1$. b) A single wall.

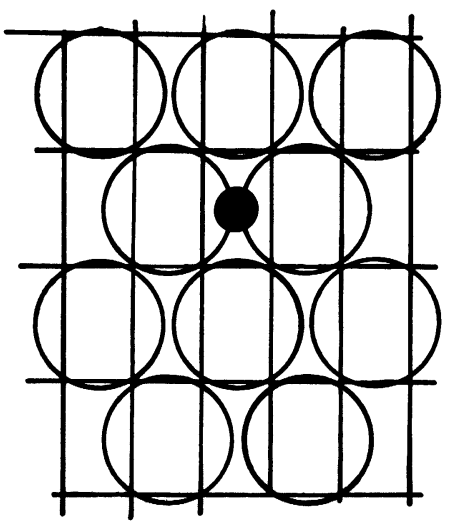

a

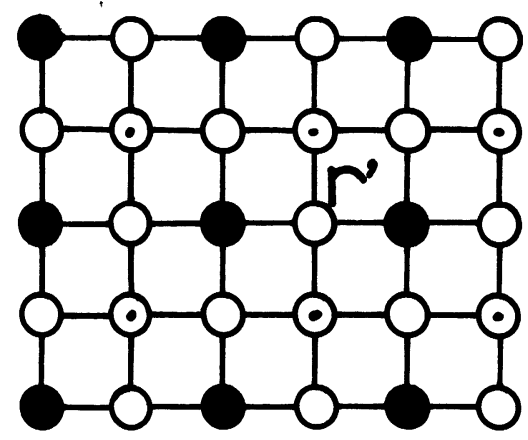

b

Fig. 2. - a) A possible mechanism for the effect of a frozen impurity (black circles) when $D=2$. The mobile atoms (empty circles, centred at the centres of the rectangles), have to choose between two sublattices. Occupation of one sublattice by the impurity favours occupation of the other sublattice by the mobile atoms. $b$ ) Decimation procedure for $d=2$. Empty circles are eliminated at the first step, dotted circles at the second step. The figure is in the $\Pi_{d}$ plane.

where the random field $H_{R}$ is equal to $H_{0}$ or $-H_{0}$ with the same probability $x$, or to 0 . This analogy with random fields in an Ising model [8-14] is not strictly generalizable to systems with more than two sublattices. However, within the rough approximation used hereafter (Section 3) more sophisticated models would yield analogous results. Therefore, we shall be contented with formula (1).

In section 2, we consider the case of a single wall (Fig. $1 b)$ characterized by its points (r, $\left.\zeta_{r}\right)$. The Hamiltonian will be written

$$
\mathscr{H}=\frac{1}{4} \sum_{r r^{\prime}} g_{r r^{\prime}}\left(\zeta_{r}-\zeta_{r^{\prime}}\right)^{2}-\sum_{r, z} H_{r}(z) S_{r}(z)
$$


where $g_{r r^{\prime}}=g$ if $r, r^{\prime}$ are neighbours

$g_{r r^{\prime}}=0$ otherwise

$$
S_{r}(z)=1 \quad \text { if } z>\zeta_{r}, \quad S_{r}(z)=-1 \quad \text { if } \quad z \leqslant \zeta_{r} .
$$

The first term in (2) expresses the tendency of the wall to be perpendicular to the $z$ axis [3]. The Hamiltonian (2) may be alternatively written as

$$
\mathscr{H}=\frac{1}{4} \sum_{r r^{\prime}} g_{r r^{\prime}}\left(\zeta_{r}-\zeta_{r^{\prime}}\right)^{2}+\sum_{r} w_{r}\left(\zeta_{r}\right)
$$

where the random function $w_{r}(\zeta)$ satisfies

$$
\overline{w_{r}(\zeta)}=0, \quad \overline{\left[w_{r}(\zeta)-w_{r^{\prime}}\left(\zeta^{\prime}\right)\right]^{2}}=\delta_{r r^{\prime}} H^{2}\left|\zeta-\zeta^{\prime}\right|
$$

where

$$
H^{2}=\overline{H_{r}^{2}}=2 x H_{0}^{2}
$$

and the bar denotes an ensemble average as in reference [15].

In order to prevent the wall to escape to infinity, the following condition will be imposed.

$$
\sum_{r} \zeta_{r}=0
$$

$\zeta_{\text {r }}$ will be treated as a continuous variable. This is always correct for $d=1(D=2)$, and this is correct for $D=3$ too, at least if the temperature is above the roughening transition $T_{R}$ or in case of disorder induced roughening [10]. The temperature $T$ will be assumed to be so low (even though than $T_{\mathrm{R}}$ ) that disorder induced fluctuations dominate temperature induced fluctuations. Dimensionality $D=d+1$ is assumed to lie between 2 and 5 .

2. Rescaling procedure. - A simple rescaling procedure is to eliminate half of the sites (Fig. $2 b$ ). Site $r^{\prime}$ can be eliminated (for each distribution of the $w_{r}^{\prime}$ 's) by treating the statistical mechanics of the $\zeta_{r}$ - dependent part in (3), namely

$$
\mathscr{H}_{r^{\prime}}=\frac{1}{2} \sum_{r} g_{r^{\prime} r}\left(\zeta_{r}-\zeta_{r^{\prime}}\right)^{2}+w_{r^{\prime}}\left(\zeta_{r^{\prime}}\right) .
$$

After $m$ steps, the number of sites is

$$
N_{m}^{\perp}=2^{-m} N^{\perp}
$$

the inter-site distance is

$$
a_{m}=2^{m / d} a
$$

and the system is assumed to be qualitatively represented by a Hamiltonian of form (3)

$$
\mathcal{H}^{(m)}=\frac{1}{2} g_{m} \sum_{\left\langle r^{\prime}\right\rangle}\left(\zeta_{r}-\zeta_{r^{\prime}}\right)^{2}+\sum_{r} w_{r}^{(m)}\left(\zeta_{r}\right)
$$

where the various $w_{r}^{(m)}$ satisfy a relation analogous to (4)

$$
\overline{\left[w_{r}^{(m)}(\zeta)-w_{r^{\prime}}^{(m)}\left(\zeta^{\prime}\right)\right]^{2}}=\delta_{r r^{\prime}} H_{m}^{2}\left|\zeta-\zeta^{\prime}\right| .
$$

Since each renormalized site corresponds to $2^{m}$ original sites,

$$
H_{m}^{2}=2^{m} H^{2} \text {. }
$$


For large $m$, the probability of $\left[w_{r}^{(m)}(\zeta)-w_{r^{\prime}}^{(m)}\left(\zeta^{\prime}\right)\right]$ is Gaussian according to the central limit theorem.

The value of $g_{m}$ is easily obtained if one writes that the contribution of each long wavelength Fourier component $\zeta_{k}$, namely

$$
g_{m}\left|\zeta_{k}\right|^{2} \sum_{r^{\prime}}\left[1-\cos \mathbf{k} \cdot\left(\mathbf{r}-\mathbf{r}^{\prime}\right)\right] \simeq g_{m}\left|\zeta_{k}\right|^{2} N_{m}^{\perp} k^{2} a_{m}^{2}
$$

is independent of $m$. Relations (8) and (9) yield :

$$
g_{m}=2^{m \frac{d-2}{d}} g
$$

A more difficult problem is to determine the free energy gain $W_{m}=N_{m}^{\perp} \bar{w}_{m}$ due to random fields at each step. It is convenient to rewrite (7) as

$$
\mathcal{H}_{r^{\prime}}^{(m)}=\hat{H}_{r^{\prime}}^{(m)}+\frac{1}{2} \gamma_{m} \zeta^{2}+\omega(\zeta)
$$

where $\zeta=\zeta_{r^{\prime}}-\bar{\zeta}_{r^{\prime}}, \bar{\zeta}_{r^{\prime}}=\sum_{a} \zeta_{a}$.

a denotes the neighbours of $r^{\prime}$,

$$
\gamma_{m}=2^{d} g_{m}, \quad \omega(\zeta)=w_{r^{\prime}}\left(\zeta_{r^{\prime}}\right)-w_{r^{\prime}}\left(\bar{\zeta}_{r^{\prime}}\right)
$$

and

$$
\hat{H}_{r^{\prime}}^{(m)}=\frac{1}{2} \sum_{a} g_{m}\left(\zeta_{a}-\bar{\zeta}_{r^{\prime}}^{2}\right)+w_{r^{\prime}}\left(\bar{\zeta}_{r^{\prime}}\right)
$$

contributes to $\mathscr{H}^{(m+1)}$. The system (14) is discussed in the Appendix A in the low temperature limit, where it is sufficient to find (for all given functions $\omega$ ) the value $\langle\zeta\rangle$ of $\zeta$ which minimizes (14), and then to calculate the ensemble average $\zeta_{m}^{2}=\overline{\langle\zeta\rangle^{2}}$. In the appendix, it is argued that an order of magnitude of $\zeta_{m}$ is obtained when the root mean square fluctuation of the last term of(14) is comparable with the second term. Using (11), one obtains

$$
\gamma_{m} \zeta_{m}^{2} \approx H_{m} \sqrt{\zeta_{m}}
$$

or, according to (12) and (13)

$$
\zeta_{m} \approx\left(H_{m} / \gamma_{m}\right)^{2 / 3} \approx 2^{m \frac{4-d}{3 d}}(H / g)^{2 / 3} .
$$

For a large sample of linear size $L=N_{\perp}^{1 / d}$, the maximum value $m_{0}$ of $m$, according to (8), is given by

$$
2^{m_{0}}=N^{\perp}=L^{d}
$$

and the total mean square displacement at site $r$ may be argued to be, for $2<d<4$

$$
\overline{\left\langle\zeta_{r}^{2}\right\rangle}=\sum_{m=0}^{m_{0}} \zeta_{m}^{2} \approx \zeta_{m_{0}}^{2} \approx L^{(8-2 d) / 3}(H / g)^{4 / 3} .
$$

The energy gain, according to (8), (11), (15)

$$
W_{m} \approx-N_{m}^{\perp}\left[\overline{\omega^{2}\left(\zeta_{m}\right)}\right]^{1 / 2} \approx-N_{m}^{\perp} H_{m} \zeta_{m}^{1 / 2} \approx-2^{2 m \frac{1-d}{3 d}} N^{\perp} H(H / g)^{1 / 3} .
$$


In the limit of a large sample the energy gain per atom :

$$
\frac{W}{N^{\perp}}=\sum_{m=0}^{\infty}\left(W_{m} / N^{\perp}\right) \approx-\frac{3 d}{d-1} H(H / g)^{1 / 3}
$$

is finite for $d>1$ or $D>2$. The divergence at the lower critical dimension

$$
D_{l}=2
$$

implies that the commensurate phase is never stable in the presence of random, frozen impurities in 2 dimensions, for short range interactions.

An alternative treatment is given in Appendix B.

3. Critical behaviour near the $\mathbf{C}-\mathbf{I}$ transition. - The picture is as in figure $1 a$. The rescaling procedure can be performed for each wall as in section 2 until $m$ reaches a value $m_{\mathrm{c}}$ for which $\zeta_{m}$ is of order $l$. This criterion is essentially that formulated by Fisher and Fisher [16] in the absence of random fields.

Relation (15) yields

$$
l \approx 2^{m_{\mathrm{c}} \frac{4-d}{3 d}}(H / g)^{2 / 3}
$$

Relation (19) is replaced by

$$
W=\frac{N^{\prime l}}{l} \sum_{m=0}^{m_{\mathrm{c}}} W_{m} \approx N \frac{w_{0}}{l}+\frac{N}{l} \frac{H}{d \cdot-1}(H / g)^{1 / 3} 2^{2 m_{\mathrm{c}} \frac{1-d}{3 d}}
$$

where $N=N^{\perp} N^{\|}$is proportional to the size of the sample. In a number of physical situations, a term $-\mu N / l$ which favours the creation of new walls. In physisorbed or intercalated systems, this term represents the effect of pressure [2,3]. Adding this term to (22) and using (21), the energy becomes

$$
W=N \frac{w_{0}-\mu}{l}+\frac{N}{d-1} H(H / g)^{\frac{d}{4-d}} l^{-\frac{6-d}{4-d}}
$$

Minimization with respect to $l$ in the physical case $D=3(d=2)$ yields a second order transition at $\mu=w_{0}$, and $1 / l$ is a linear function of $\mu$ in the incommensurate phase near the C-I transition.

$$
\frac{1}{l} \sim\left|\mu-w_{0}\right|
$$

At constant $\mu, 1 / l$ can be shown to be a linear function of $T$ near the C-I transition temperature $T_{\mathrm{c}}$

$$
\frac{1}{l} \sim\left|T_{\mathrm{c}}-T\right| \text {. }
$$

4. Discussion - There is some analogy between our model and an Ising model in a random field, as can be seen from formulae (1) and (2). In the Ising model, however, $\zeta_{r}$ is not a singlevalued function of $\mathbf{r}$. Our formula (20) is in contrast with the result $D_{l}=3$ obtained by other authors [10-13] for the Ising model in a random field, but it is not clear whether this implies a disagreement with these authors. Also, our result (17) is in contrast with formula (2) of Binder et al. [12].

If one wants to describe an interface in an Ising model with random fields by formula (3), the variations $\left(\zeta_{r}-\zeta_{r^{\prime}}\right)$ are found to be small for small $H$, so that the harmonic approximation in (3) 
is apparently consistent. The interface energy is found to be positive for small $H$ at low $T$ for $D>2$, and this suggests $D_{l}=2$ for the Ising model as in the old paper of Imry and Ma [8] (see also Ref. [14]). We do not wish to make any definite statement about the Ising model in random field, but we argue in the Appendix A that the replica trick [17] used by some authors [10, 11] may be difficult to handle. Of course, if there is a transition, it cannot be a single continuous one [9]. The arguments presented here are for $T=0$ but the extension to low temperature is easy and does not modify the results. More detailed calculation will be published later.

This work was partly motivated by experimental work [18] on 3-D systems according to which $1 / l \sim \sqrt{\left|T-T_{c}\right|}$. We have no explanation of this result, which is in disagreement with (25).

Acknowledgments. - We were greatly helped by a preprint of T. Nattermann on the same subject. However, our conclusions are different. We are grateful to the K. F. A. Jülich for an invitation to a stay where we had illuminating discussions with Prof. K. Binder. Discussions with D. Mukamel and T. Ziman are also acknowledged.

Note added in proof. - After completing this work, we were informed of a very similar one by C. Grinstein and S. K. Ma (preprint, april 1982). These authors criticize the replica trick as we do in Appendix A (but in more detailed way) and present a calculation which is just technically different from our section 2 . They recover, in particular, our formulae (17) and (20). They do not consider the C-I transition as we do in sections 1 and 3. Also, our Appendix A presents a particularly simple case where the replica trick is difficult to handle.

Appendix A. - The last two terms of (14) are

$$
\hat{H}=\frac{1}{2} \gamma \zeta^{2}+\omega(\zeta)
$$

where

$$
\omega(\zeta)=-\sqrt{l} \sum_{z=-\infty}^{\infty} H(z) \frac{z-\zeta}{|z-\zeta|}
$$

$z$ is an integer multiple of the arbitrary infinitesimal constant $l$. The $H(z)$ 's are independent random variables. For definiteness their probability may be assumed Gaussian though less restrictive assumptions may be used. Their definition is achieved with the relations

$$
\overline{H(z)}=0 ; \quad \overline{H(z) H\left(z^{\prime}\right)}=H^{2} \delta_{z z^{\prime}} .
$$

It is convenient to introduce a reduced length $x$ and a reduced Hamiltonian $\nVdash$

$$
\begin{aligned}
& x=\zeta(\gamma / H)^{2 / 3} \\
& \varkappa=\frac{1}{H}(\gamma / H)^{1 / 3} \hat{H} .
\end{aligned}
$$

$\nVdash$ has the universal form

$$
\mathcal{K}=\frac{1}{2} x^{2}-\sqrt{l^{\prime}} \sum_{\xi=-\infty}^{\infty} h(\xi) \frac{\xi-x}{|\xi-x|}
$$

where

$$
h(\xi)=\frac{1}{H} H\left(\xi[\gamma / H]^{2 / 3}\right)
$$


satisfies the universal law

$$
\overline{h(\xi) h\left(\xi^{\prime}\right)}=\delta_{\xi \xi^{\prime}}
$$

and $l^{\prime}=l(\gamma / H)^{2 / 3}$, so that $\xi$ is an integer multiple of the infinitesimal quantity $l^{\prime}$. Relations (A.2) and (A.8) might of course be written in integral form.

The universal form of (A.8) and (A.6) implies that all dimensionless functions $P(\zeta)$ of physical interest depend on $\gamma, H$ and $\zeta$ only through the product $x=\zeta(\gamma / H)^{2 / 3}$. The quantity of interest here is the probability $P_{0}(\zeta)$ that the minimum of (A.1) lies between $-\zeta$ and $\zeta$. Relations (15) and (16) are easily deduced from the statement that $P_{0}(\zeta)$ is a function of $\zeta(\gamma / H)^{2 / 3}$ alone.

Since the replica trick is often used $[10,11]$ to solve similar problems, it is of interest to apply it to Hamiltonian (A.1). The second term can be written as in section $1: \omega(\zeta)=\sum H(z) S(z)$ where

$$
S(z)=(z-\zeta) /|z-\zeta|
$$

standard methods [17] yield the replica Hamiltonian

$$
\tilde{\mathscr{C}}=\frac{1}{2} \gamma \sum_{\alpha} \zeta_{\alpha}^{2}+\beta H^{2} \sum_{\alpha \gamma}\left|\zeta_{\alpha}-\zeta_{\gamma}\right| .
$$

The replica trick consists in applying the identity

$$
\left\langle\zeta^{2}\right\rangle=\lim _{n \rightarrow 0} \frac{1}{n} \sum_{\alpha} \overline{\left\langle\zeta_{\alpha}^{2}\right\rangle Z^{n}}
$$

We have not been able to treat the limit $n \rightarrow 0$. One difficulty is that quantity (A.10) is analytic in $H$ for all integers $n \geqslant 1$, while the limit $\zeta_{m}^{2}$ for $n \rightarrow 0$ is not analytic as shown by (15). This suggests that the average value (A.10) is non-analytic in $n$ at $n=0$ (or somewhere between 0 and 1) and that the replica trick is difficult to handle.

Appendix B. - A heuristic approach of Hamiltonians (2) and (3) consists in replacing them by

$$
\mathscr{H}_{\mathrm{cff}}=\sum_{k}\left[\frac{1}{2} g k^{2}\left|\zeta_{k}\right|^{2}-h_{-k} \zeta_{k}\right]
$$

where $\zeta_{k}$ is the Fourier transform of $\zeta_{r}$ and $h_{k}$ is a Gaussian random field. At $T=0$

$$
\begin{aligned}
\left\langle\zeta_{k}\right\rangle & =h_{k} / g k^{2} \\
\overline{\left\langle\zeta_{r}^{2}\right\rangle} & =\frac{1}{N} \sum_{k} \overline{\left\langle\zeta_{k}\right\rangle^{2}} \approx \int_{k_{\mathrm{c}}} \overline{\left|h_{k}\right|^{2}} \frac{k^{d-1} \mathrm{~d} k}{g^{2} k^{4}} \\
& \approx \overline{\left|h_{k_{\mathrm{c}}}\right|^{2}} k_{\mathrm{c}}^{d-4} / g^{2}
\end{aligned}
$$

where $k_{\mathrm{c}}=1 / L . \overline{\left.h_{k_{\mathrm{c}}}\right|^{2}}$ is given by the following self-consistency requirement derived from (4)

$$
\begin{aligned}
H^{2} \overline{\left\langle\zeta_{r}^{2}\right\rangle^{1 / 2}} & \approx \overline{w_{r}^{2}} \approx \frac{1}{N^{1}} \sum_{k} \overline{\left|h_{k}\right|^{2}} \overline{\left\langle\left|\zeta_{k}\right|\right\rangle^{2}} \\
& \left.\approx \int \overline{\left(\left|h_{k}\right|^{2}\right.}\right)^{2} k^{d-1} \mathrm{~d} k / g^{2} k^{4} \\
& \left.\approx \overline{\left(\left|h_{k_{\mathrm{c}}}\right|^{2}\right.}\right)^{2} k_{\mathrm{c}}^{d-4} / g^{2}
\end{aligned}
$$


$\overline{\left|h_{k_{c}}\right|^{2}}$ and $\overline{\left\langle\zeta_{r}^{2}\right\rangle}$ can be determined from (B.2) and (B.3). The results are formula (17) and

$$
\overline{\left|h_{k}\right|^{2}} \approx H^{2}(g / H)^{2 / 3} k^{(4-d) / 3} \text {. }
$$

In the above calculation it is assumed that $1<d<4$.

\section{References}

[1] McMillan, W. L., Phys. Rev. B 14 (1976) 1496.

[2] VIllain, J., in Ordering in strongly fluctuating systems, Ed. T. Riste (Plenum Press, New York) 1980, p. 214.

[3] Haldane, F. D. M., Villain, J., J. Physique 42 (1981) 1673.

[4] Schulz, H. J., Phys. Rev. Lett. 46 (1981) 1685.

[5] Nattermann, T., J. Physique 43 (1982) 631.

[6] Nelson, D. R., Halperin, B. I., Phys. Rev. B 19 (1979) 2457.

[7] Glachant, A., Jaubert, M., Bienfait, M., Boato, G., Surf. Sci. 115 (1981) 219.

[8] ImRY, Y., MA, S., Phys. Rev. Lett. 35 (1975) 1399.

[9] Young, A. P., J. Phys. C 10 (1977) L 257.

[10] Pytte, E., Imry, Y., Mukamel, D., Phys. Rev. Lett. 46 (1981) 1173.

[11] Mukamel, D., Pytte, E., preprint (1981).

[12] Binder, K., Imry, Y., Pytte, E., Phys. Rev. B 24 (1981) 6736.

[13] Kogon, H. S., Wallace, D. J., J. Phys. A 14 (1981) L 527.

[14] Shapir, Y., Aharony, A., J. Phys. C 15 (1982) 1361.

[15] Sherrington, D., J. Phys. C 8 (1975) L 208.

[16] Fisher, M. E., Fisher, D., Phys. Rev. B 25 (1982) 3192.

[17] Edwards, S. F., Anderson, P. W., J. Phys. F 5 (1975) 965.

[18] Moudden, A. H., Thèse, Faculté d'Orsay (1980). 